\title{
Practical Applications of the Large-Eddy Simulation Technique for Wind Environment Assessment around New National Stadium, Japan (Tokyo Olympic Stadium)
}

\author{
Takanori Uchida1*, Ryo Araya ${ }^{2}$ \\ ${ }^{1}$ Research Institute for Applied Mechanics (RIAM), Kyushu University, Fukuoka, Japan \\ ${ }^{2}$ Environmental GIS Laboratory Co. Ltd, Fukuoka, Japan \\ Email: ^takanori@ riam.kyushu-u.ac.jp
}

How to cite this paper: Uchida, T. and Araya, R. (2019) Practical Applications of the Large-Eddy Simulation Technique for Wind Environment Assessment around New National Stadium, Japan (Tokyo Olympic Stadium). Open Journal of Fluid Dynamics, 9, 269-291.

https://doi.org/10.4236/ojfd.2019.94018

Received: September 11, 2019

Accepted: October 27, 2019

Published: October 30, 2019

Copyright $\odot 2019$ by author(s) and Scientific Research Publishing Inc. This work is licensed under the Creative Commons Attribution International License (CC BY 4.0).

http://creativecommons.org/licenses/by/4.0/

\begin{abstract}
In this study, we developed a new computational fluid dynamics (CFD) model called Airflow Analyst that deepens the affinity between CFD and geographic information system (GIS). First, a precise simulation of the surface-mounted cube was conducted. Validation testing based on the obtained data confirmed the predictive accuracy of Airflow Analyst. Second, New National Stadium Japan (Tokyo Olympic Stadium) was accurately reproduced in a computer, capturing the latest detailed urban area data for the base. For the target of the constructed 3D models, simulations with a large number of grid points/cells (CFD) were conducted. These simulations reproduced the complex turbulent flow fields both inside and outside the stadium. The experiment successfully reproduced the CFD simulation using a large number of grid points/cells, where the conditions of the wind flow ventilation from the sky were similar to those of the intended stadium design.
\end{abstract}

\section{Keywords}

CFD, LES, GIS, Surface-Mounted Cube, New National Stadium Japan

\section{Introduction}

Urban environments in our society are affected by the various impacts of wind, such as strong wind damage and ventilation. Thus, the built environment in urban design and regional planning needs to be assessed and optimized. Every step during the examination process for plans requires the involvement of citizens, administrators, and developers. Our research team was engaged in software de- 
velopment for urban planning and architecture design, focusing on new computational fluid dynamics (CFD) [1] with a high affinity for geographic information system (GIS) [2]. Recent research results in the fields we are interested in have been reviewed in the literature [1]. We have been developing Airflow Analyst [3] [4] [5] [6], which is extension software for ArcGIS, which is versatile GIS software. The biggest advantage of Airflow Analyst is that users are able to analyze the flow field characteristics or passive scalar transport and diffusion in regions with arbitrarily shaped objects without considering landform features or building clusters.

Airflow Analyst, running on the GIS system, has the following advantages:

1) Large labor savings in the preparation of three-dimensional (3D) data for urban areas due to its usage of geospatial information resources, which are circulated free-of-charge or sold;

2) Large-scale reductions in constructing $3 \mathrm{D}$ data planning due to the integration of computer-aided design (CAD) and building information modeling (BIM);

3) Instant simulation with intuitive operation due to the visual confirmation of scales on maps for computational wind directions, domains, and grid generation;

4) Visualizing the computational results of $3 \mathrm{D}$ data on a map and storing geographic information-spatial references by coordinates, thereby enabling superpositions of spatial analysis with other spatial information; and

5) The availability to distribute and share using superpositions with web mapping services.

Conventional wind-flow simulations for urban areas require specialized knowledge and skills, as well as enormous amounts of time and labor to create 3D models and generate grids integrating local terrain], building shapes, and design plans. To reflect wind-flow simulations in a plan or a design, however, a system with simple and intuitive operation is required to examine data immediately after being obtained. This system should reduce the work involved in the above CFD analysis, and planners should be able to appropriately perform numerical simulations.

Governments and open data communities, both inside and outside Japan, are rapidly and increasingly providing free 3D data management. Therefore, geospatial information infrastructures have been established with 3D urban data. Specifically, BIM and Construction Information Modeling (CIM) are employed, with enhanced data compatibility with GIS. BIM and CIM have also enabled 3D data usage in design planning. Along with this, advanced techniques have been developed to extract data from satellite and aerial photographs, as well as photogrammetric image data acquired by unmanned aerial vehicles (UAVs). A new data creation method has been proposed: point cloud data obtained via laser surveying can generate minute $3 \mathrm{D}$ data, and all these data can be analyzed via integration with the GIS.

Airflow Analyst is the first software program in the world to complete the process from grid generation to the visualization of data computation results. 
The present report demonstrates the first validation test of the predictive accuracy of Airflow Analyst for a surface-mounted cube. Second, this paper introduces a reproduction of the New National Stadium Japan (Tokyo Olympic Stadium) using 3D topography Advanced World 3D Map (AW3D) data [7], which were created from a combination of high-resolution images taken by satellite photographs. Then, we report large-scale numerical simulation cases for wind flow. Other analyses developed using Airflow Analyst are described in the Appendix.

\section{Summary of the Airflow Analyst Software}

For the numerical simulations, we used the Airflow Analyst software package, for which a collocated grid in a general curvilinear coordinate system was adopted. In this collocated grid, the velocity components and pressure are defined at the grid cell centers, and variables that result from multiplying the contravariant velocity components by the Jacobian are defined at the cell faces. For the numerical technique, we adopted the finite difference method (FDM), and a large-eddy simulation (LES) model was used for the turbulence model. In the LES model, a spatial filter was applied to the flow field to separate eddies of various scales into the grid-scale (GS) components, which are larger than the computational grid cells, and sub-grid scale (SGS) components, which are smaller than the computational grid cells. Large-scale eddies, i.e., the GS components of turbulence eddies, are directly numerically simulated without the use of a physically simplified model. In contrast, dissipation of energy, which is the main effect of small-scale eddies, i.e., the SGS components, is modeled according to a physics-based analysis of the SGS stress.

For the equations governing the flow, a filtered continuity equation for incompressible fluid (Equation (1)) and a filtered Navier-Stokes equation (Equation (2)) are used. When passive scalar transport and diffusion were considered, the standard convection-diffusion equation for a passive scalar was solved with the linkage of the above equations of flow field characteristics.

Since we investigated wind fields with mean wind speeds of $5-10 \mathrm{~m} / \mathrm{s}$, the effects of the vertical thermal stratification of the atmosphere (atmospheric stability) were negligible. For the computational algorithm, a method similar to a fractional step (FS) method [8] was used, and a time marching method based on the Euler explicit method was adopted. Poisson's equation for pressure was solved using the successive over-relaxation (SOR) method. To discretize all the spatial terms except for the convective term in Equation (2), a second-order central difference scheme was applied. For the convective term, a third-order upwind difference scheme was applied. The interpolation technique by Kajishima [9] was used for the fourth-order central differencing that appears in the discretized form of the convective term. For the weighting of the numerical diffusion term in the convective term discretized by third-order upwind differencing, $\alpha=0.5$ was used instead of $\alpha=3.0$ from the Kawamura-Kuwahara scheme 
[10] to minimize the influence of numerical diffusion. For LES SGS modeling, the standard Smagorinsky model [11] was adopted with a model coefficient of 0.1 , in conjunction with a wall-damping function (Equations (3)-Equation (8)).

$$
\begin{gathered}
\frac{\partial \bar{u}_{i}}{\partial x_{i}}=0 \\
\frac{\partial \bar{u}_{i}}{\partial t}+\bar{u}_{j} \frac{\partial \bar{u}_{i}}{\partial x_{j}}=-\frac{\partial \bar{p}}{\partial x_{i}}+\frac{1}{\operatorname{Re}} \frac{\partial^{2} \bar{u}_{i}}{\partial x_{j} \partial x_{j}}-\frac{\partial \tau_{i j}}{\partial x_{j}} \\
\tau_{i j} \approx \overline{u_{i}^{\prime} u_{j}^{\prime}} \approx \\
\frac{1}{3} \overline{u_{k}^{\prime} u_{k}^{\prime}} \delta_{i j}-2 v_{S G S} \bar{S}_{i j} \\
v_{S G S}=\left(C_{s} f_{s} \Delta\right)^{2}|\bar{S}| \\
|\bar{S}|=\left(2 \bar{S}_{i j} \bar{S}_{i j}\right)^{1 / 2} \\
\bar{S}_{i j}=\frac{1}{2}\left(\frac{\partial \bar{u}_{i}}{\partial x_{j}}+\frac{\partial \bar{u}_{j}}{\partial x_{i}}\right) \\
f_{s}=1-\exp \left(-z^{+} / 25\right) \\
\Delta=\left(h_{x} h_{y} h_{z}\right)^{1 / 3}
\end{gathered}
$$

\section{Validation Testing of the Prediction Accuracy of the Airflow Analyst Software}

Here, the results of validation testing are reported for the prediction accuracy of the airflow with a surface-mounted cube. Qualitative and quantitative assessments were conducted using flow visualization and turbulent flow measurement with a split-film anemometer. Figure 1 shows a bird's eye view of the thermally stratified wind tunnel used for the present study. Figure 2 shows a side view of the surface-mounted cube's setting state. Figure 3 depicts a close-up view of the surface-mounted cube in the wind tunnel's test section.

As shown in Figure 4, the U-shaped horseshoe vortex surrounding a surface-mounted cube is apparent [12]. In the present simulation, a horseshoe vortex was reproduced with a 3D structure, as shown in Figure 5. Figure 5 visualizes the flow by using the smoke-wire technique in the wind tunnel experiment, which used the flow field in the vicinity of the surface-mounted cube. We observed that a highly complex flow field formed in the vicinity of the surface-mounted cube. Figure 6 provides the results of the simulations. Comparing both images shown in Figure 6 and Figure 8, Figure 6 demonstrates reproductions of flow patterns similar to the wind tunnel experimental result in Figure 7. Figure 8 shows the streamline image for the time-average flow field of the simulations. Observing this result, a standing vortex formed behind the surface-mounted cube in the time-average flow field. At the measurement station set in the center of the vortex (indicated by the black line in the figure), we focus on a graph comparing the vertical profile of $U$-velocity obtained from the 


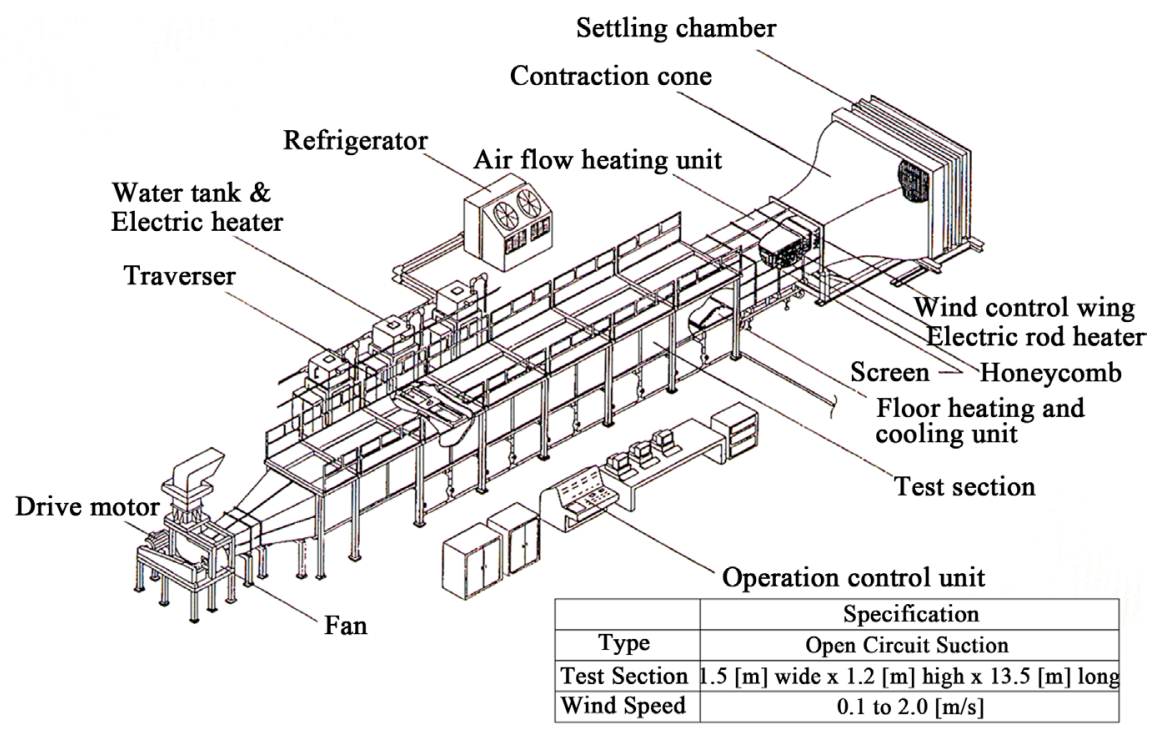

Figure 1. Summary of the thermally stratified wind tunnel.

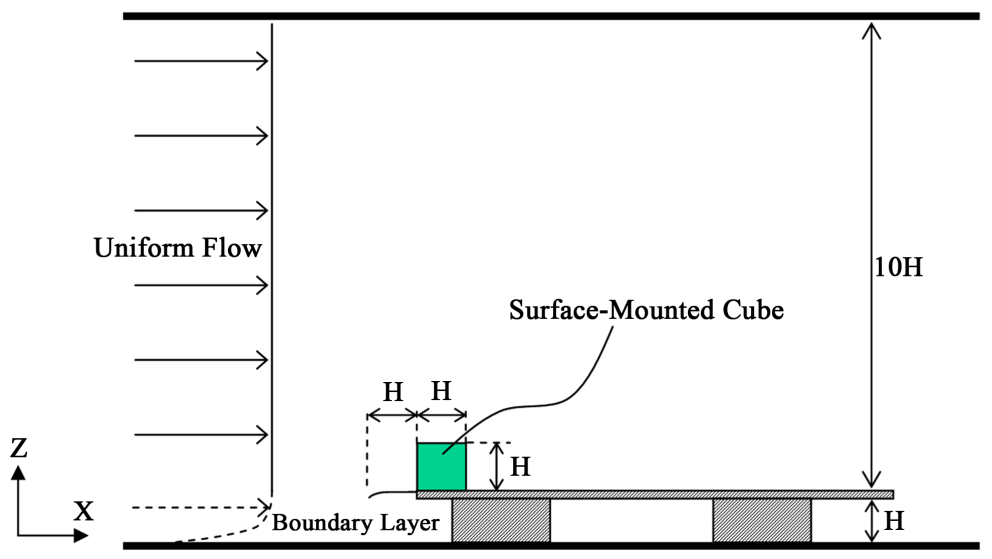

Figure 2. Side view of the wind tunnel experiment.

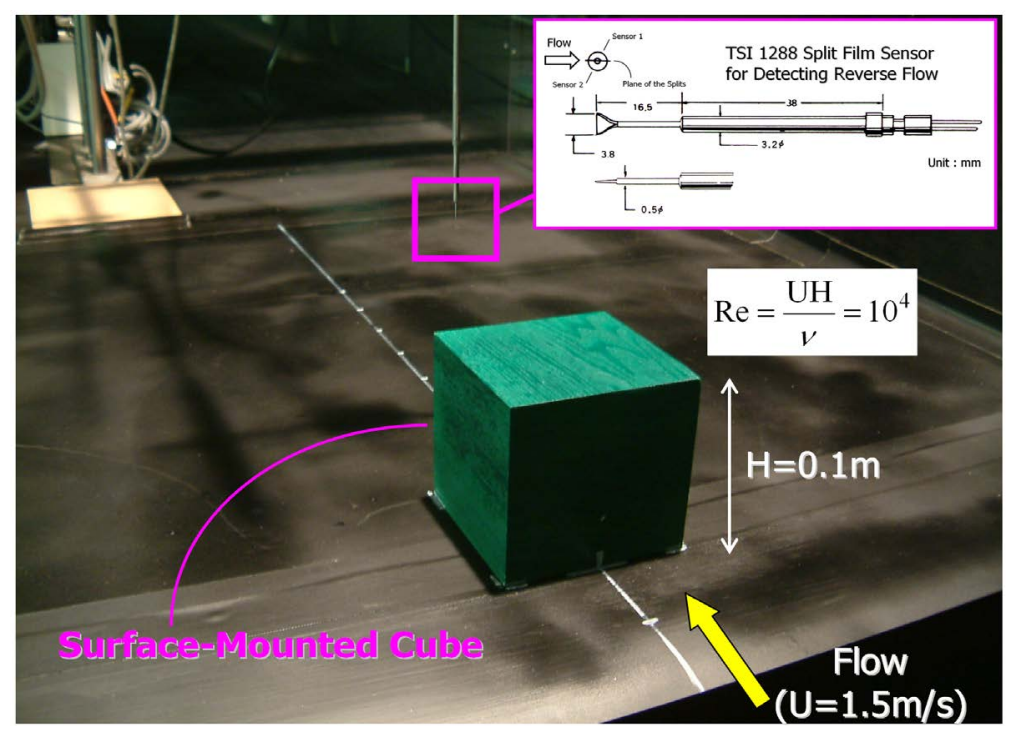

Figure 3. Close-up view of the wind tunnel experiment. 


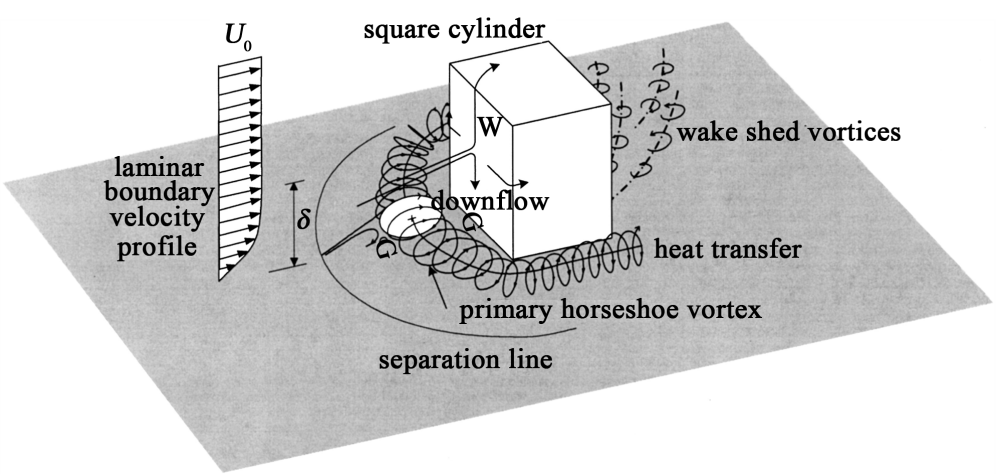

Figure 4. Schematic view of the flow around a surface-mounted cube (square cylinder) [12].

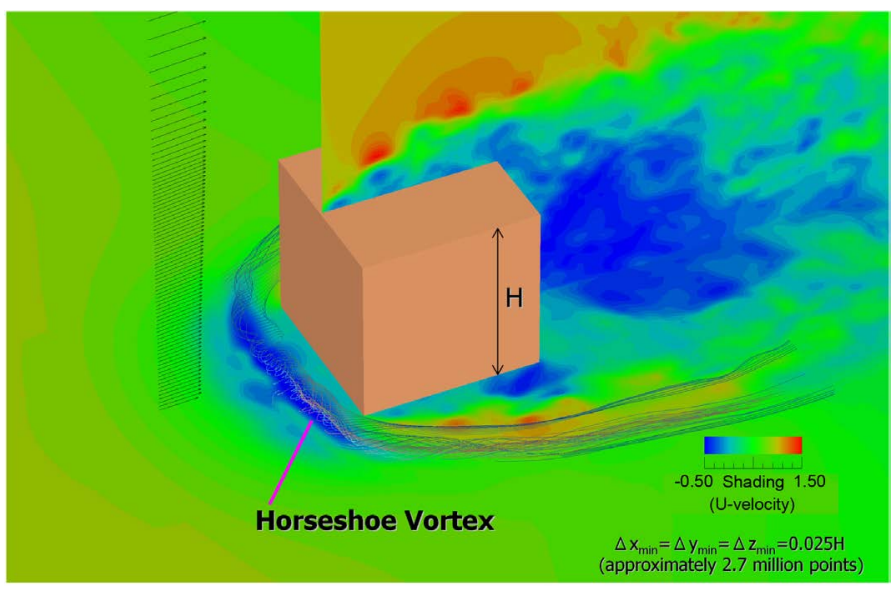

Figure 5. Horseshoe vortex reproduced in the present large-eddy simulation (LES). Instantaneous flow field.

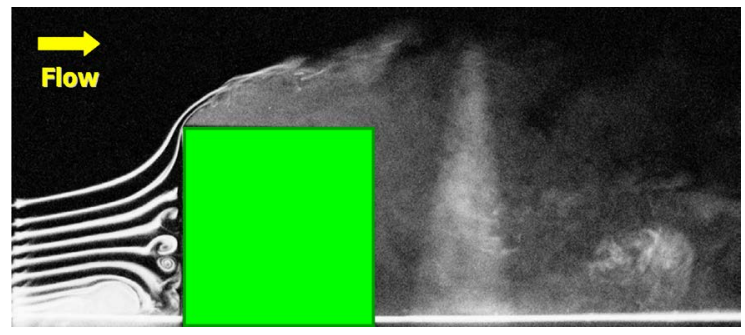

(a)
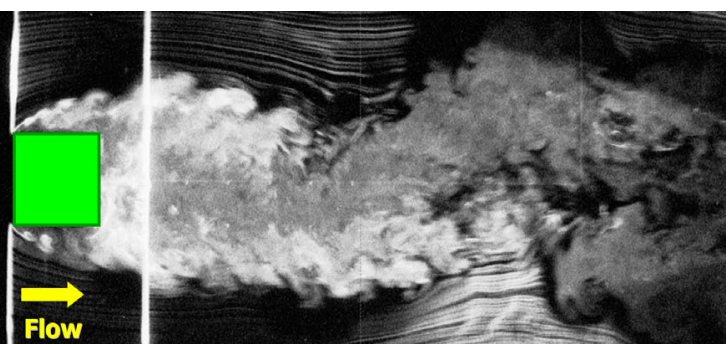

(b)

Figure 6. Flow visualization in the wind tunnel experiment by using the smoke-wire technique; instantaneous flow field: (a) side view, $y=0$ and (b) top view, $\mathrm{z}=0.5 \mathrm{H}$. 


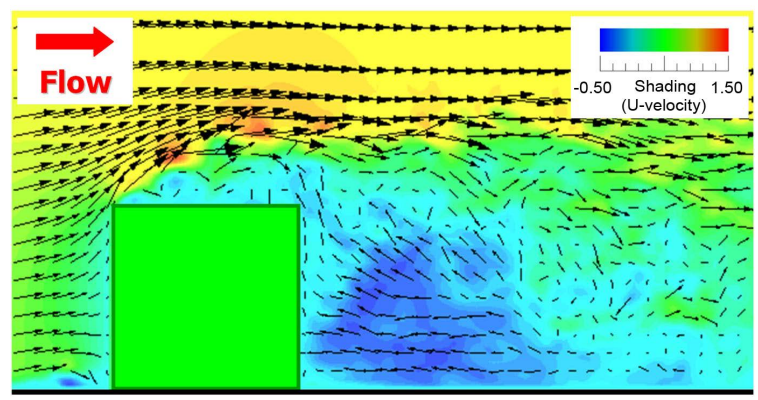

(a)

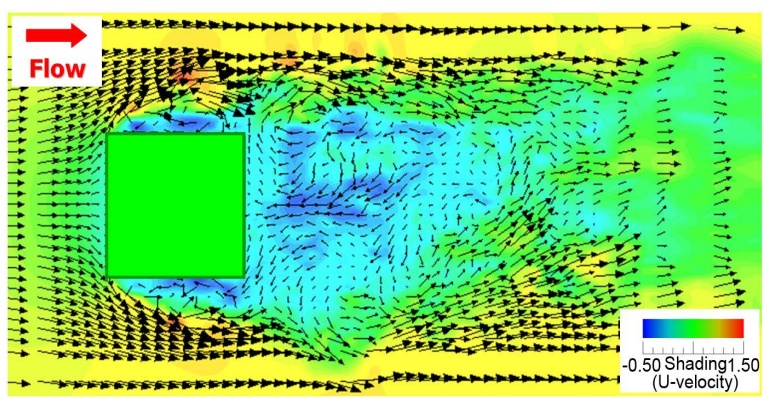

(b)

Figure 7. Flow visualization in the numerical simulation; instantaneous flow field: (a) side view, $\mathrm{y}=0$ and $(\mathrm{b})$ top view, $\mathrm{z}=0.5 \mathrm{H}$.
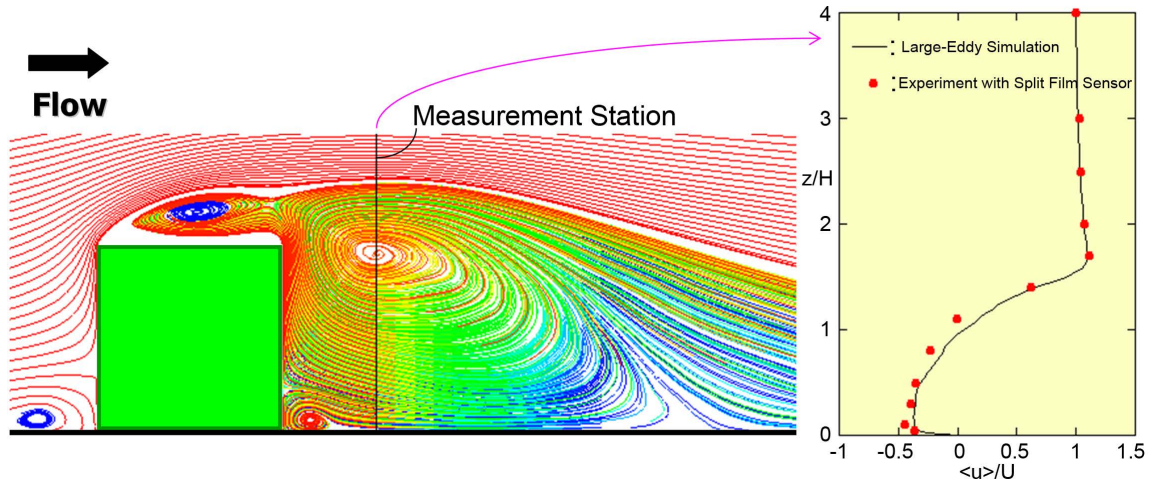

Figure 8. Streamline for the time-averaged flow field, $y=0$, and comparison with the U-velocity profile.

present numerical simulation with those from the wind tunnel experiment. Satisfactory agreement was found between the results in the wind tunnel experiment and the simulations. Judging from these results, the effectiveness of Airflow Analyst was objectively confirmed.

\section{Application of Airflow Analyst Software to More Realistic and Complex Situations}

Next, we introduce simulations of the New National Stadium Japan (Tokyo Olympic Stadium). The results of this elaborate reproduction were obtained by CFD simulation with a large number of grid points/cells using the latest detailed data for urban areas. 


\subsection{Structured Mesh Generation Based on GIS Technique}

Generally, four steps are required in conventional software to perform CFD simulations of urban areas, as shown in Figure 9. Process 1, inputting the 3D building data, requires considerable operating time. Here, we will explain the construction method of 3D modeling (and other) of the New National Stadium Japan (Tokyo Olympic Stadium).

The Advanced World 3D Map (AW3D) data [7] were employed to express locations and the height of landscape characteristics, buildings, and trees. AW3D [7] is a 3D map created by imagery data from multiple high-resolution satellites in the USA. These data combine city images photographed from various angles without dead angles. Therefore, this technology can distinguish even a building or a tree with 3D images. The map reproduces accurate urban area configurations, providing individual height differences for a building whose roof shape is not horizontal. AW3D data provide global coverage, covering even area information where map data are difficult to obtain. The provided data are categorized into digital terrain models (DTM) for ground surface height with a five-meter resolution, architectural polygons for building shapes, and tree polygons for tree shapes. These data are provided in GIS file formats (tagged image file format (TIFF) format and the ESRI Shapefile format) by the universal transverse Mercator coordinate system (UTM). No processing is needed to directly use these data for numerical wind simulations.

We originally created 3D data based on the published planning drawings of the shape of the Olympic Stadium using 3D modeling software, Sketch UP. The 3D model, which we created, was input to the GIS according to the position and scale of the UTM coordinate system. Airflow Analyst can identify 3D model data from DIM (TIFF), ESRI Shapefile, CAD, and BIM. Then, the software enables mesh generation with ease. Consequently, planners are free from the laborious input operation used in preparing a wind flow analysis and are able to focus on the examination of computational results and reflect on the improvement of a proposed plan.

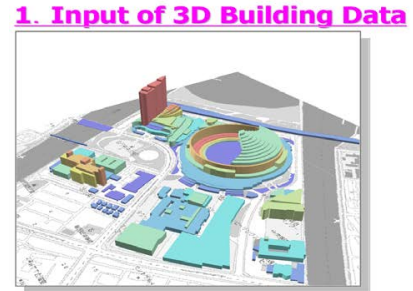

2. Mesh Generation

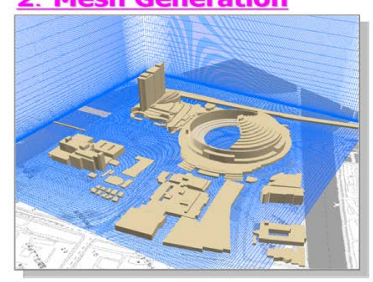

1, 2) CFD Pre-Processing, 3) CFD Solver,

4) CFD Post-Processing

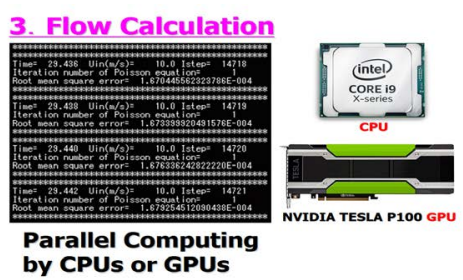

4. Flow Visualization

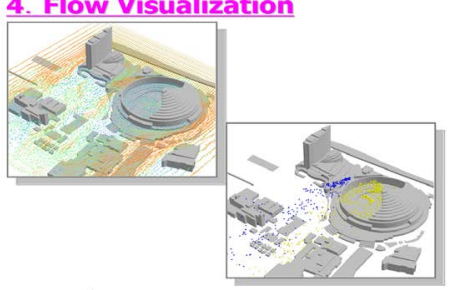

Figure 9. Procedure for the computational fluid dynamics (CFD). 
In this study, two types of CFD simulations were conducted for the New $\mathrm{Na}$ tional Stadium Japan (Tokyo Olympic Stadium). One case is for the stadium only. The other is a case where all the surrounding factors, such as geographic features in the vicinity of the stadium, buildings around the stadium, and surface roughness are taken into account in addition to the stadium. Through these comparisons, the purpose of this study is to clarify the effect of the urban area around the New National Stadium Japan (Tokyo Olympic Stadium).

Figure 10 depicts a 3D model of the computer reproduction, consisting of the research target, the New National Stadium Japan (Tokyo Olympic Stadium), and the surrounding factors. Figure 11 shows the computational domain and the other parameters set in this study. An enlarged view of the computational grid around the New National Stadium Japan is provided in Figure 12.

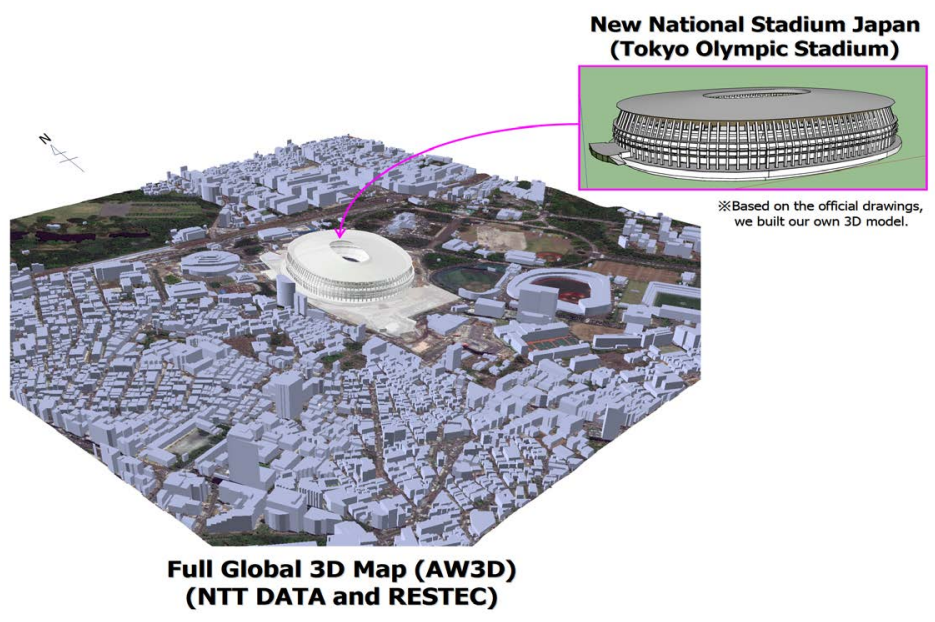

Figure 10. New National Stadium Japan created based on AW3D and surrounding three-dimensional (3D) models.

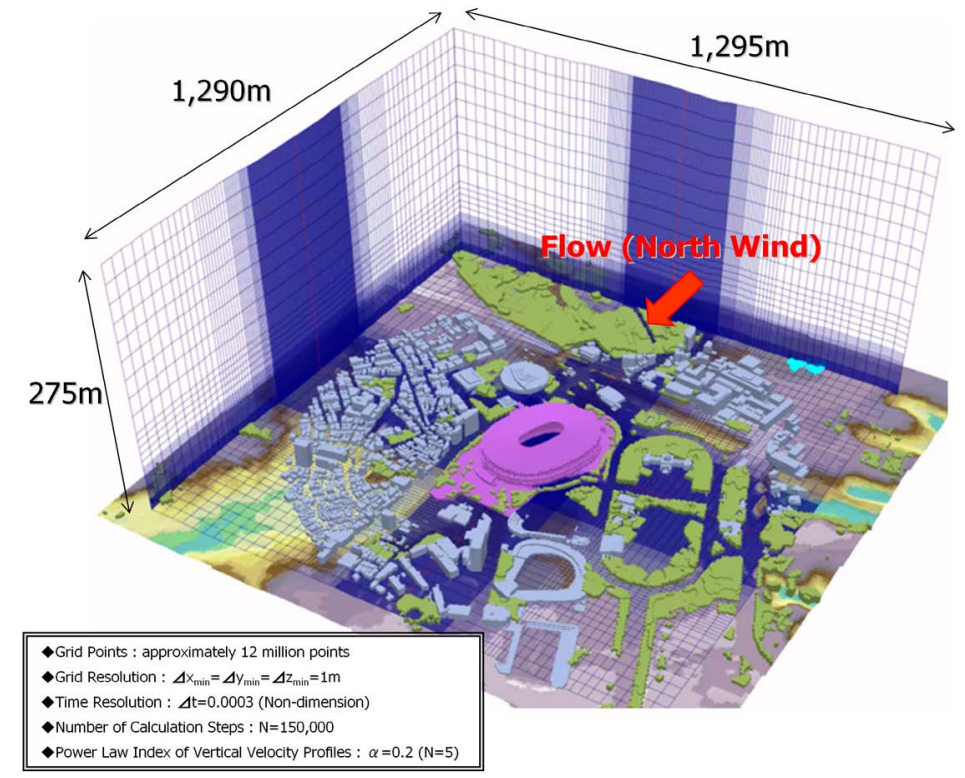

Figure 11. Computational domain and grid information. 


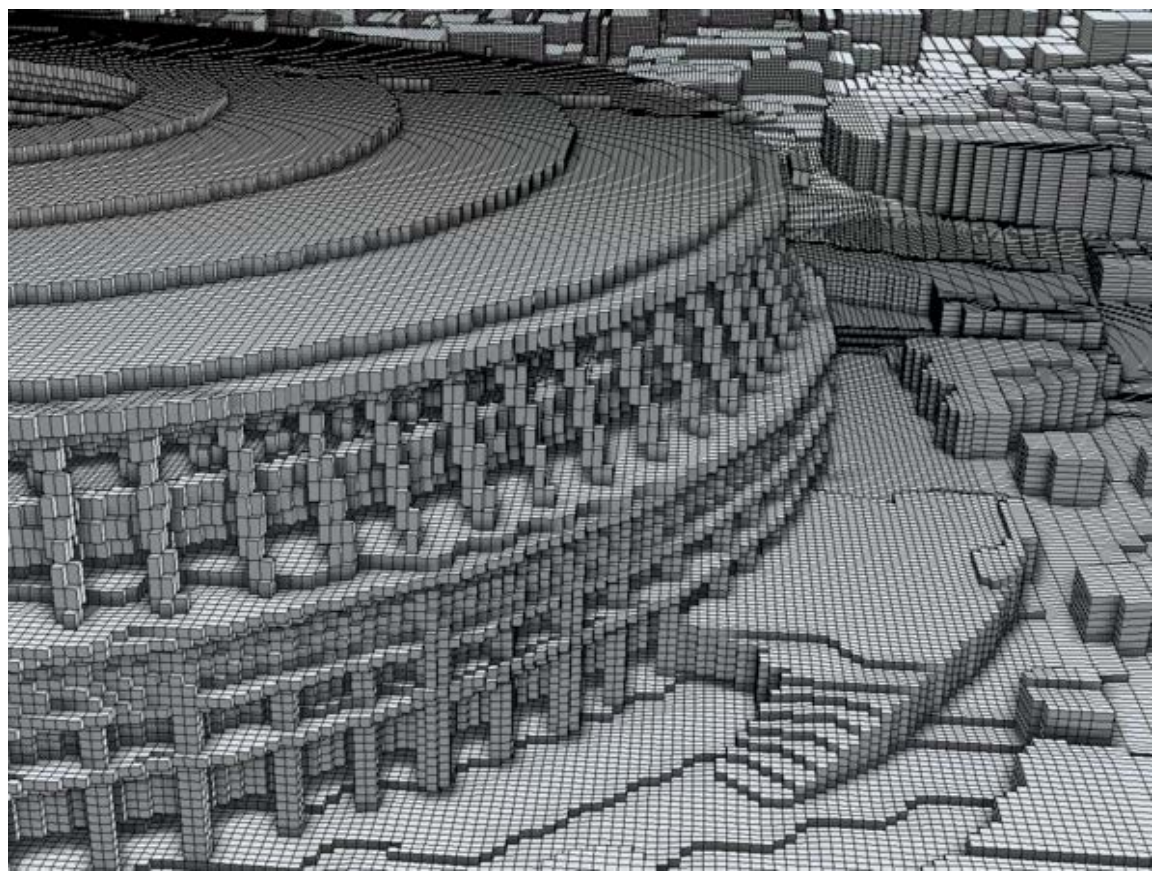

Figure 12. Enlarged view of the grid around the New National Stadium Japan.

\subsection{Numerical Results and Discussions}

Figure 13 shows the air flow pattern in the vicinity of the ground surface (at a height of five meters). The target of the image in Figure 13(a) is only the stadium; it does not consider topography, building, or surface roughness, whereas Figure 13(b) does. That is to say, Figure 13(b) is the result of a simulation that is closer to reality. The simulation results in Figure 13(a), where only the stadium was considered, indicate that a wide variety of strong wind fields form in the side-peripheries. Wind speed was accelerated locally $1.2-1.3$ faster than the approaching wind speed (the region with color ranging from yellow to red in Figure 13). Accordingly, we recognized that the wind flow field, which formed inside the stadium (the blue region in Figure 13) showed complex changes. We confirmed that a complex wind flow field formed on the downstream side of the New National Stadium Japan (Tokyo Olympic Stadium). Focusing on Figure 13(b), where the topography, buildings, and surface roughness in the vicinity of the stadium were examined, we confirmed large reductions in the abovementioned sequence, such as the formation of a strong and complex wind field.

Next, we discuss the flow visualization in the vertical plane of the Tokyo Olympic Stadium. As shown in Figure 14, natural and effective wind ventilation is accomplished with unique devices in the upper parts of the stadium, as expected (see the red circle) [13]. The results of the simulations that we conducted (Figure 15) confirmed that both of the stadium reproductions efficiently took in air from the sky, although there were slight differences in the angles where wind from the sky was taken into the stadium from the upper to the lower portions. We are currently conducting a more quantitative evaluation of the difference in the angle at which the air above is taken into the stadium. 

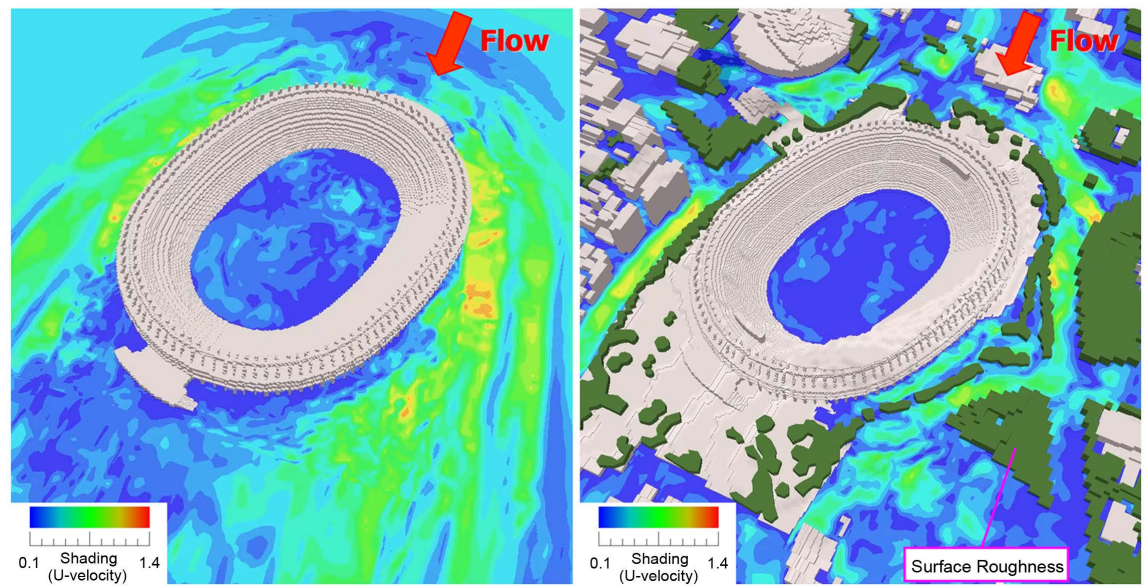

Figure 13. Flow visualization of the numerical simulation near the ground, instantaneous flow field: (a) only the stadium case and (b) case with considering topography, buildings, and surface roughness.

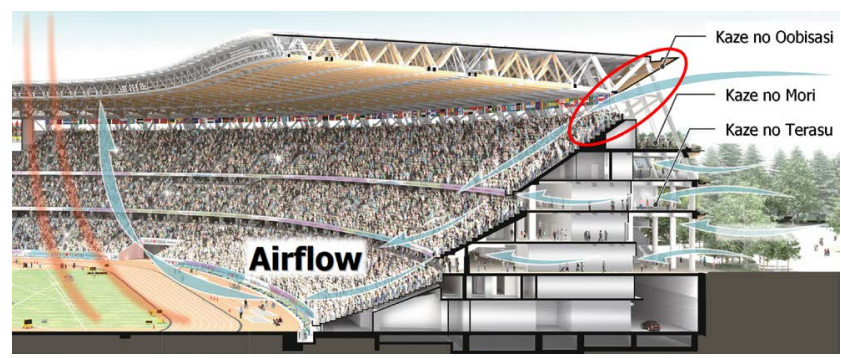

Figure 14. Image of the stadium cross section [13].

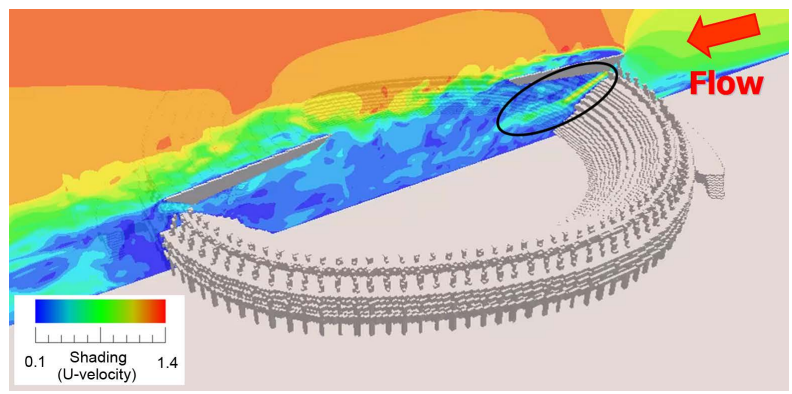

(a)

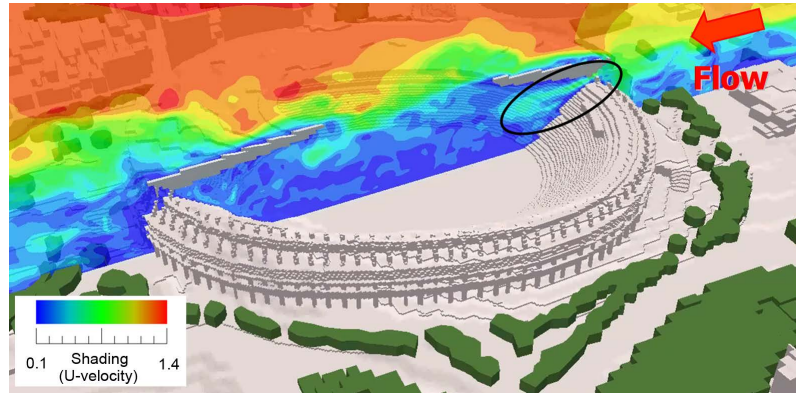

(b)

Figure 15. Flow visualization in the numerical simulation in the vertical plane. Instantaneous flow field: (a) only the stadium case and (b) case with considering topography, buildings, and surface roughness. 


\section{Conclusions}

In this study, we developed a new computational fluid dynamics (CFD) model called Airflow Analyst that deepens the affinity between CFD and geographic information system (GIS). Here, we demonstrated the promising effectiveness of Airflow Analyst as follows. First, a precise simulation of the surface-mounted cube was conducted. Validation testing based on the obtained data confirmed the predictive accuracy of Airflow Analyst. Second, New National Stadium Japan (Tokyo Olympic Stadium) was accurately reproduced in a computer, capturing the latest detailed urban area data for the base. For the target of the constructed 3D models, simulations with a large number of grid points/cells (CFD) were conducted. These simulations reproduced the complex turbulent flow fields both inside and outside the stadium. The experiment successfully reproduced the CFD simulation using a large number of grid points/cells, where the conditions of the wind flow ventilation from the sky were similar to those of the intended stadium design. Thus, these results objectively confirm the effectiveness of Airflow Analyst, which we have been developing.

The government of Japan is currently facilitating the fifth Science and Technology Basic Plan ("Society 5.0") as a vision for future society. This Society 5.0, which represents the fifth form of society in our human history, follows the hunting society, agricultural society, industrial society, and information society. Society 5.0 means a new era, taking advantage of artificial intelligence (AI), robots, and the Internet of things (IoT), to create new values and services. At present, the Smart City Project, which focuses on this era of Society 5.0, is being developed in many directions. Leveraging leading-edge AI and IoT technology, the mission of the Smart City Project is to increase quality of life by efficiently managing basic life infrastructure and services with environmental considerations. This new type of city is designed with the aim of continuous economic development (Figure 16). Among administrators' roles, there are programs for

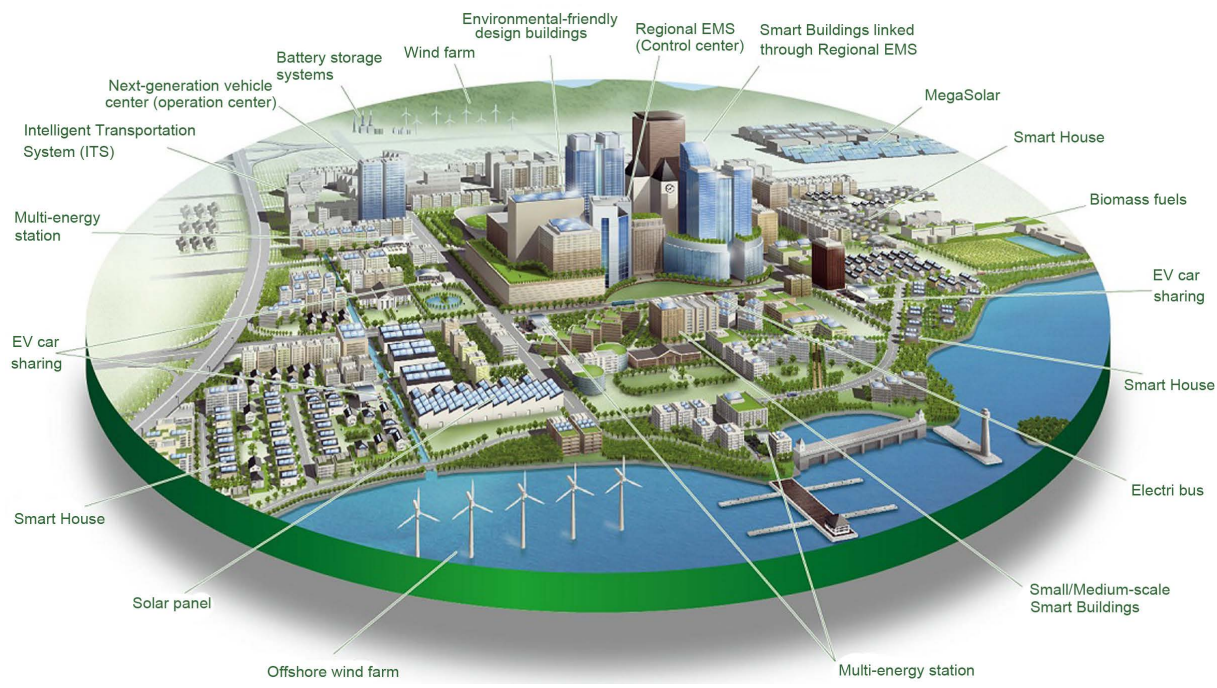

Figure 16. Smart city concept [14]. 
Smart Governance, where the common data infrastructure, which is the foundation of economic activities and community developments, is accessed online based on GIS. Both public and private sectors will collaborate to provide and share city data to ensure decision making is based on transparency and trust among all administrators, planners (experts), and citizens. This system aims to improve the quality of life of all cities in our society. Airflow Analyst contributes by providing simulation analysis for city planning based on an assessment of the wind environment (comfort), an analysis of local strong winds (eddies of winds around high buildings), an assessment of suitable wind energy sites, and the ability to analyze smell and gas diffusion. Airflow Analyst is advantageous for the integrating data from Smart Governance and sharing simulation analyses. We will continue to develop Airflow Analyst, which is a promising analysis platform for this Smart City Project.

\section{Acknowledgements}

For conducting this research, the authors were provided with the various types of data by NTT DATA and RESTEC. The authors also received significant cooperation from: 1) Kyushu University New Campus Planning Office; 2) the Institute of Astronomy (IoA), the University of Tokyo; 3) the Housing and Urban Planning Bureau, Fukuoka City Government; 4) The Japan Aerospace Exploration Agency (JAXA); and 5) the Inter-university Research Institute Corporation Research Organization of Information and Systems, National Institute of Polar Research. The authors would like to express their gratitude to all the organizations.

\section{Conflicts of Interest}

The authors declare no conflicts of interest regarding the publication of this paper.

\section{References}

[1] Toparlar, Y., Blocken, B., Maiheu, B. and van Heijst, G.J.F. (2017) A Review on the CFD Analysis of Urban Microclimate. Renewable and Sustainable Energy Reviews, 80, 1613-1640. https://doi.org/10.1016/j.rser.2017.05.248

[2] https://www.esri.com

[3] Saito, T., Uchida, T. and Araya, R. (2013) Computation of Airflow for Space Rocket Launch. Reports of Research Institute for Applied Mechanics, 145, 121.

[4] Uchida, T. and Araya, R. (2012) Huge Computation of Buoyant Gas Diffusion in the Real City Area. Reports of Research Institute for Applied Mechanics, 142, 63.

[5] Uchida, T. and Araya, R. (2012) Huge Computation of Airflow around the Fukuoka City. Reports of Research Institute for Applied Mechanics, 142, 55.

[6] Araya, R. (2011) Wind Environment Design Using Wind Modeling. ESRI Map Book, Vol. 26, ESRI Press, Redlands, 86.

[7] https://www.aw3d.jp

[8] Kim, J. and Moin, P. (1985) Application of a Fractional-Step Method to Incompres- 
sible Navier-Stokes Equations. Journal of Computational Physics, 59, 308-323. https://doi.org/10.1016/0021-9991(85)90148-2

[9] Kajishima, T. (1994) Upstream-Shifted Interpolation Method for Numerical Simulation of Incompressible Flows. Bulletin of the Japan Society of Mechanical Engineers B, 60, 3319. https://doi.org/10.1299/kikaib.60.3319

[10] Kawamura, T., Takami, H. and Kuwahara, K. (1986) Computation of High Reynolds Number Flow around a Circular Cylinder with Surface Roughness. Fluid Dynamics Research, 1, 145-162. https://doi.org/10.1016/0169-5983(86)90014-6

[11] Smagorinsky, J. (1963) General Circulation Experiments with the Primitive Equations: I. Basic Experiments. Monthly Weather Review, 91, 99-164. https://doi.org/10.1175/1520-0493(1963)091<0099:GCEWTP>2.3.CO;2

[12] https://ascelibrary.org

[13] https://www.jpnsport.go.jp/newstadium

[14] https://medium.com 


\section{Appendix}

Airflow analysis, which is continuing to develop, is used for analyses in diverse fields. Here, we introduce some results from previous studies. First is the case of Shiki Hall of Ito Campus, Kyushu University, which was built in the center zone of the campus (Figure A1). We elaborated upon an investigation of wind environment with a one-meter spatial resolution (Figure A2). This hall is a circular

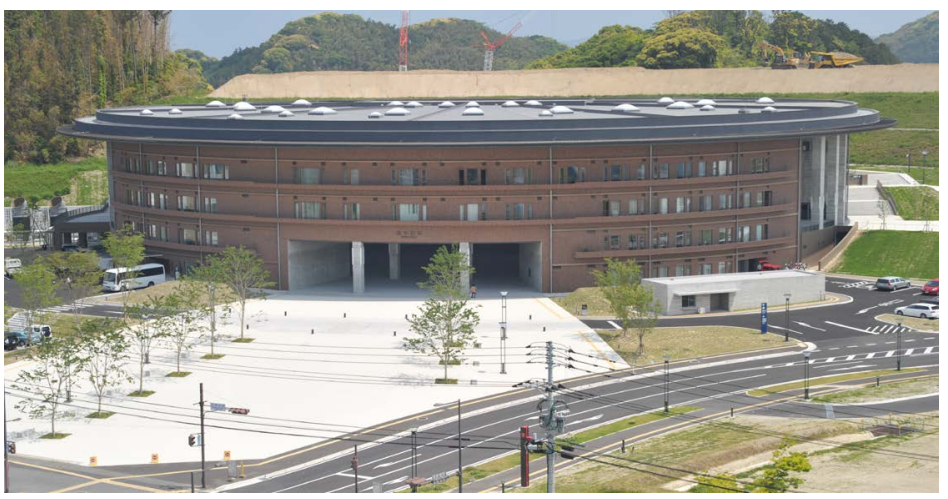

Figure A1. Shiiki Hall at Ito Campus, Kyushu University, Japan.

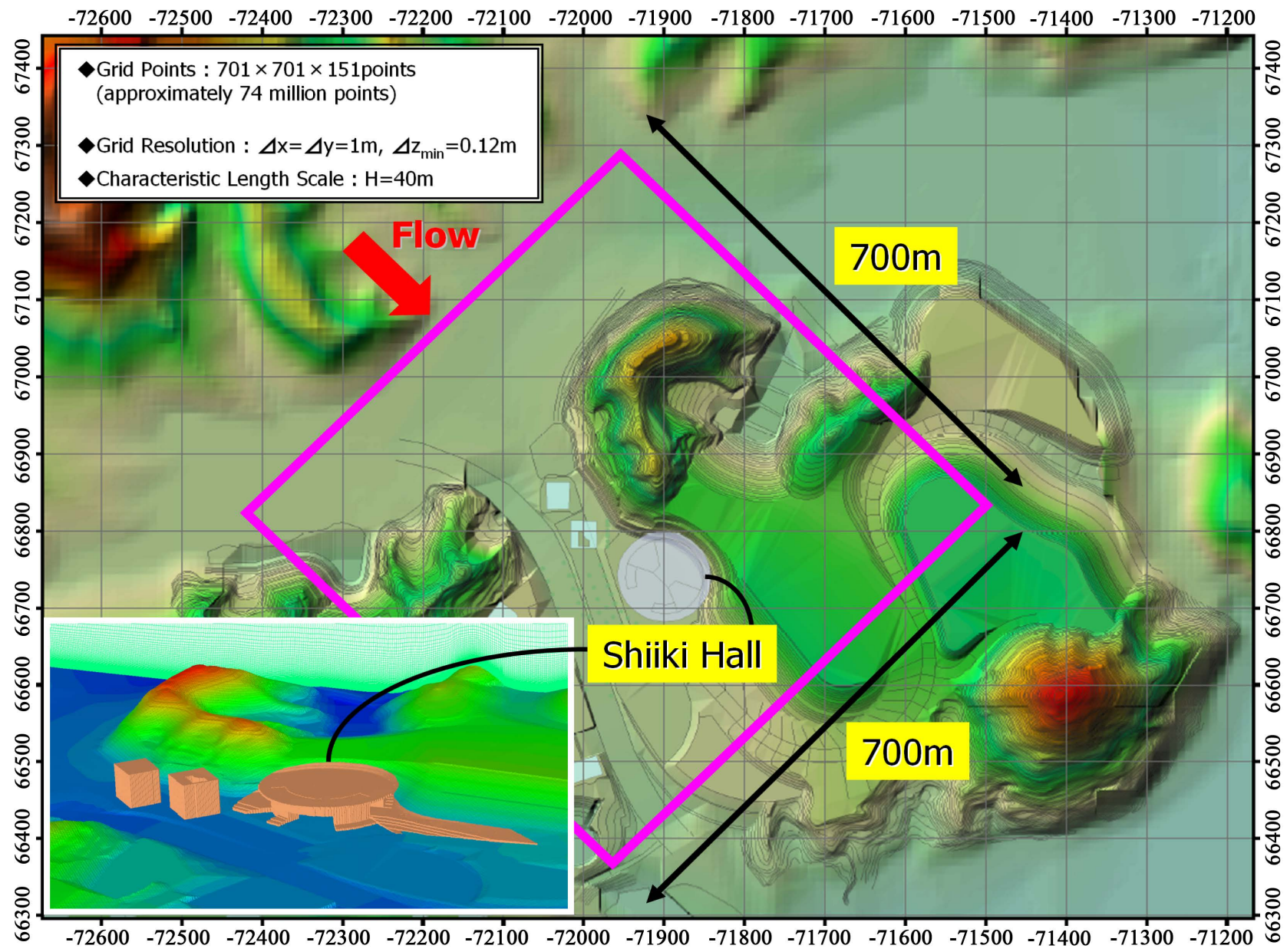

Figure A2. Computational domain and grid information. 
building with a diameter of $100 \mathrm{~m}$ and consists of a main hall and an administrative building. Its maximum capacity is 3000 people, and various events are held there, such as entrance ceremonies, degree conferment ceremonies, academic conferences, and other large-scale events. The wind flow environment of this hall was assessed at the request of the Planning and Promotion Division of New Campus, Kyushu University in 2012. Focusing on a huge half-cylindrical shaped open space, which was called the Galleria, this building's wind environment was examined to determine if severe local wind occurs. Approximately 74 million grids were generated for this wind flow simulation. The computational results were analyzed in detail, assuming cases with a northwest wind whose occurrence frequency is high during winter. This analysis confirmed that a strong wind region was not generated inside the Galleria (Figure A3(A)).

Next, a simulation result for Fukuoka, Japan is reported. The simulation shown in Figure A4, which was performed in 2011, reproduces the complex air flow behavior generated in the urban Fukuoka area, creating individual building models of building shape clusters, with an approximately three-meter spatial resolution. The findings of this study were thought to be able to effectively use wind energy as an option for renewable energy. Specifically, preliminary calculations were provided for wind energy resources and to guide site selections for wind turbines. The findings could contribute to urban development planning in harmony with future wind environments in Fukuoka, suggesting methods to improve the wind environment (heat island countermeasures and other measures).

The Urban Reconstruction Section, Urban Development, and Promotion Department, Housing and Urban Planning Bureau, Fukuoka City Government asked us to conduct a computer simulation of the wind flow for the urban $\mathrm{Fu}$ kuoka area (Tenjin and Hakata Station areas) in 2010-2011. Based on the basic city planning surveys with building data in the current situation, individual building shapes in the Tenjin and Hakata Station areas were accurately simulated with a four-meter spatial resolution, and tree shapes were also simulated using aerial photography. Figure A5 provides one example of the simulation

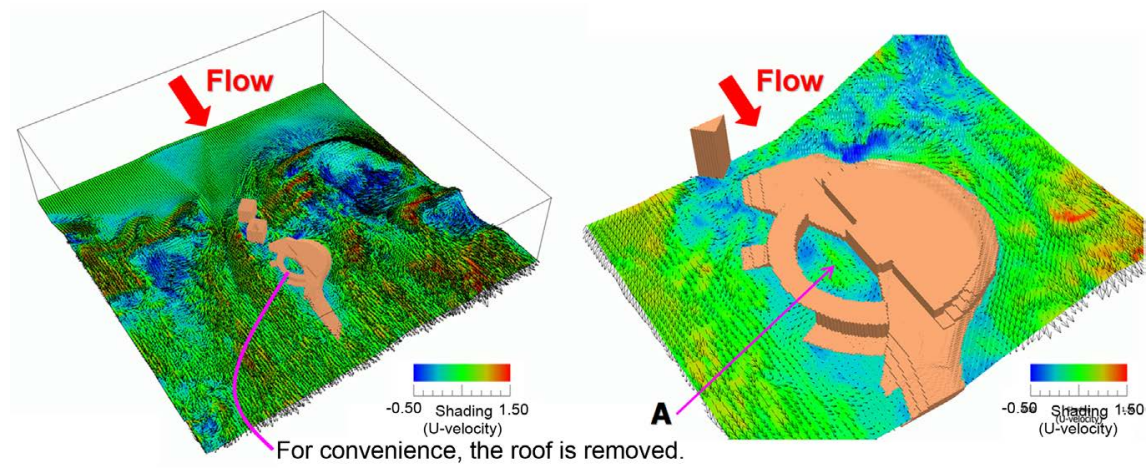

Figure A3. Flow visualization of the numerical simulation near the ground, instantaneous flow field: (a) overall and (b) close-up view. 


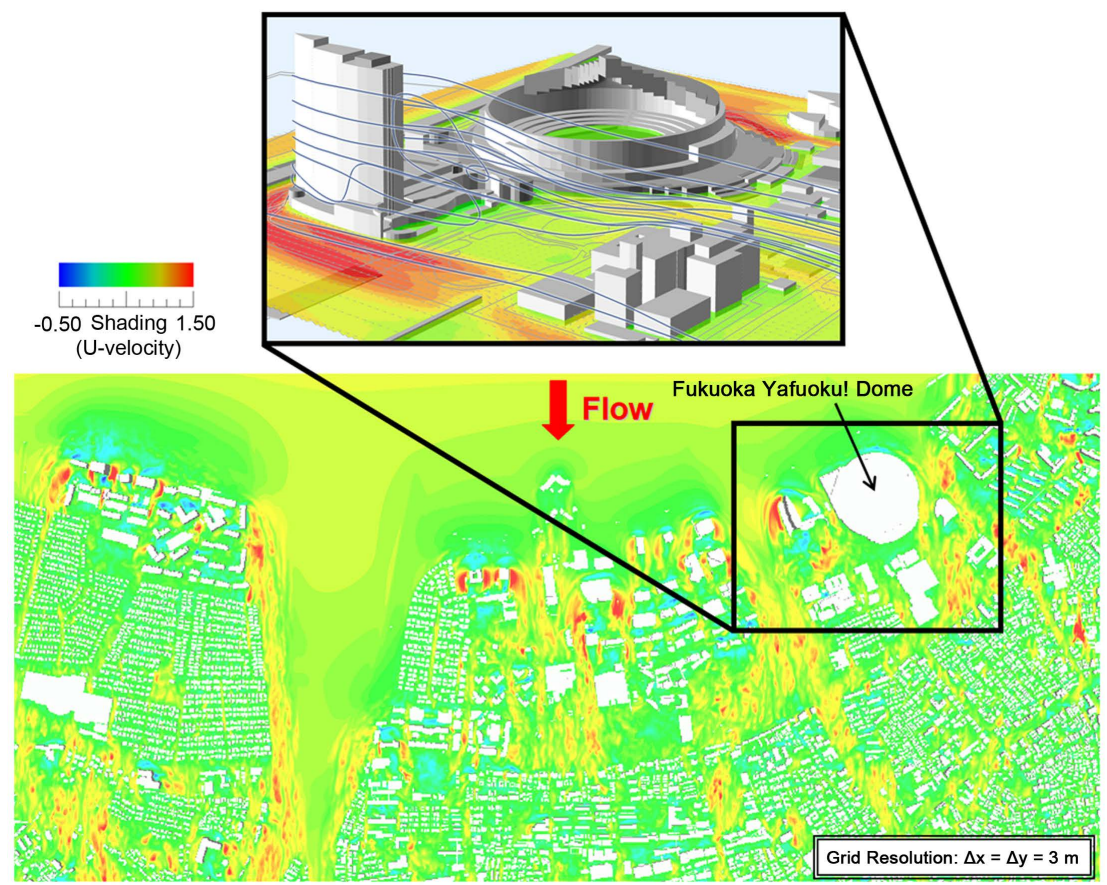

Figure A4. Flow visualization in the numerical simulation near the ground. Instantaneous flow field.

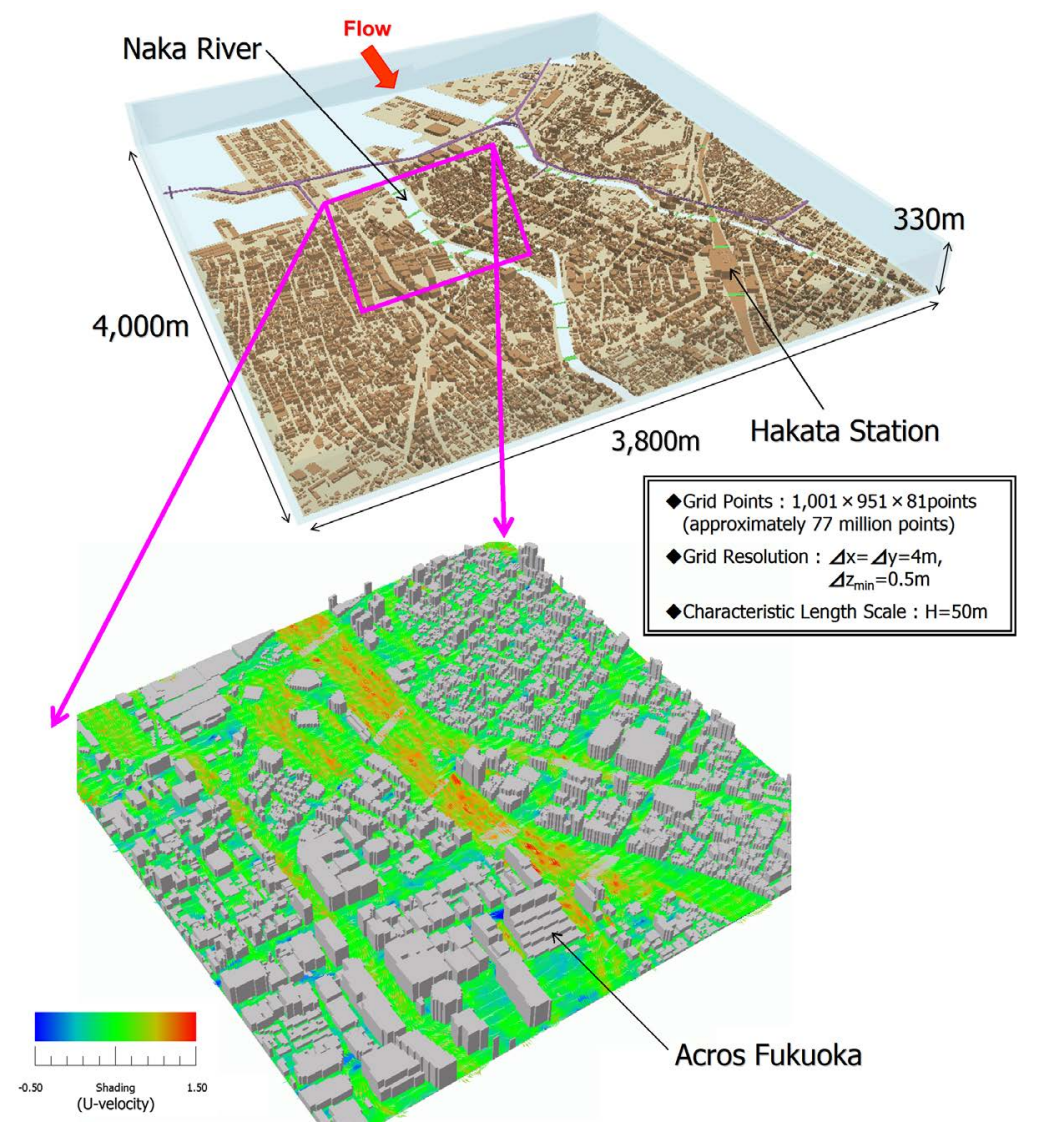

Figure A5. Flow visualization in the numerical simulation near the ground, instantaneous flow field in Fukuoka, Japan. 
results, with a northwest wind as the target. Focusing on the vicinity of the Naga River, the river was found to bed a path for the wind. The wind that intruded from Hakata Bay increased its speed by 1.3 - 1.4 times and intruded into the center of the city, as was demonstrated. Visual confirmation suggested that, contrarily, the Acros Fukuoka building hindered the intrusion of the accelerated wind turbulence to some extent. The findings could contribute to the urban development planning in harmony with future wind environments in Fukuoka, suggesting improvements for the wind environment (heat island countermeasures). The findings of our study could enable the mapping of strong winds due to typhoons, as well as developing simulations for pollen diffusion.

Here, we introduce the study findings of a joint research project (November 2012-March 2016) with the Japan Aerospace Exploration Agency (JAXA). In this project, we investigated wind flows with objects from the Tanegashima Space Center and Uchinoura Space Center, employing CFD simulation with a large number of grid points/cells. First, to verify the predictive accuracy of Airflow Analyst, we performed a simulation to reproduce wind tunnel experiments that had been previously conducted (Figure A6). As a result, we clarified that our numerical wind flow simulation could highly accurately reproduce the previous wind tunnel experiments (Figure A7).

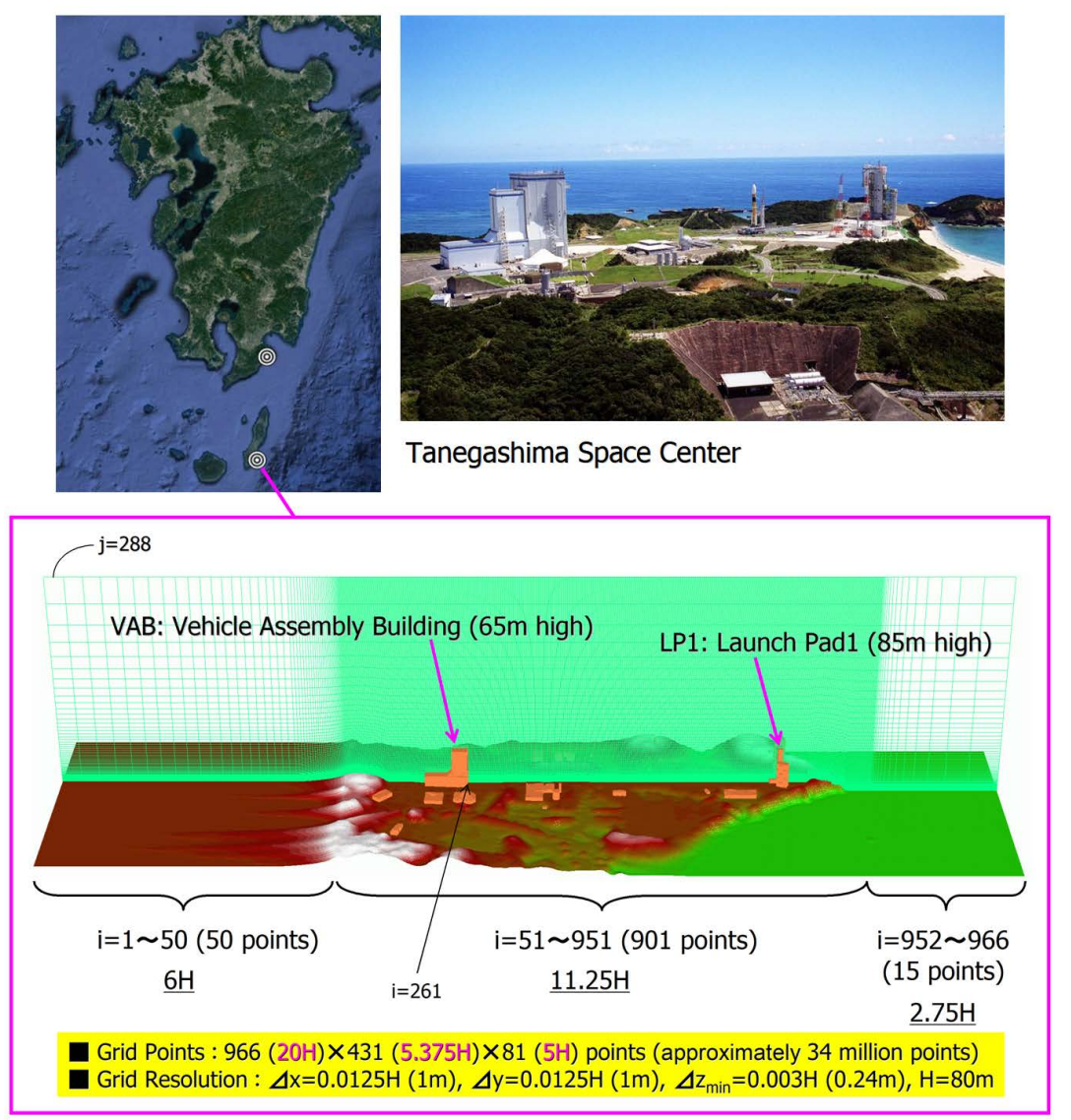

Figure A6. Calculation domain and calculation parameters for Tanegashima Space Center. 


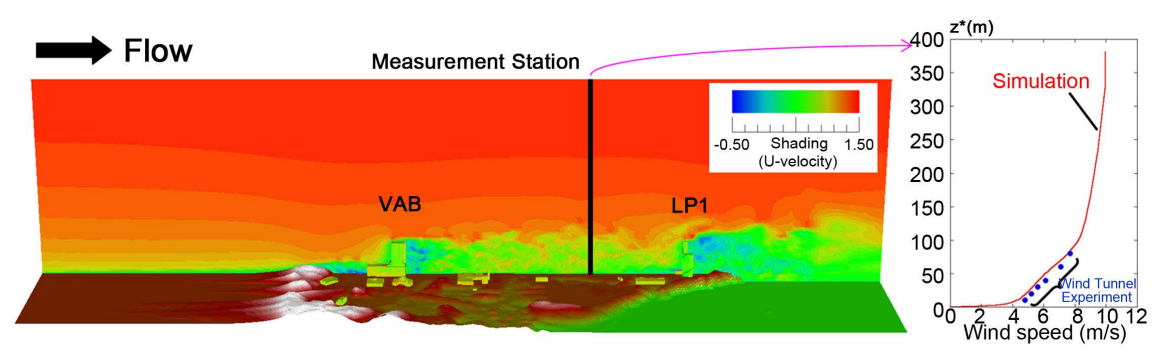

Figure A7. Flow visualization of the numerical simulation in the vertical plane, instantaneous flow field.

To support the Epsilon Launch Vehicle, we conducted a CFD simulation with a large number of grid points/cells with the Uchinoura Space Center as a target. The wind direction of the target was northwest, which was recognized as the direction of the prevailing wind during the winter. The results of the numerical analysis clarified that when the northwest wind blew for a long period, the area around Uchinoura Space Center was severely affected by the separated flow from Mt. Kunimi-yama (887 m elevation) located on the upstream side of the center (Figure A8). We expect further analyses of the obtained computational results to be used for the improvement of wind speed monitoring at the rocket launch site.

In Figures A9-A11, we introduce simulations of the University of Tokyo Atacama Observatory (TAO) Project (August 2014-March 2016), at the request of the University of Tokyo. TAO is a project to construct a $6.5 \mathrm{~m}$ infrared-optimized telescope at the summit of Cerro Chajnantor (5640 m elevation), located in the Atacama Desert in Northern Chile, and to promote astronomical observations that aim to elucidate the origins of the Galaxy and the planets. This project is led by the Institute of Astronomy (IoA) at the University of Tokyo, Japan.

First, a numerical wind flow condition simulation was performed for a vast area, with the Cerro Chajnantor as the subject, assessing the strength and the vertical distribution of the wind intruding into the Tokyo Atacama Observatory (TAO, Figure A10). Second, TAO was accurately reproduced with a $0.15 \mathrm{~m}$ spatial resolution, and an enormous amount of numerical data were obtained from the CFD simulation with a large number of grid points/cells. By analyzing the enormous number of numerical data obtained from the large-scale simulation, we successfully reproduced the changes in complex air flows, which formed inside and outside the telescope's dome (Figure A11). The number of computational grid points was approximately 100 million in this simulation. We will continue to develop this research to examine the obtained numerical data. We plan to establish a system to control the airflow inside the dome systematically.

The last case that we introduce in this study is a joint investigation between the Inter-University Research Institute Corporation Research Organization of Information and the Systems and National Institute of Polar Research from February 2014 to March 2016. The research object for this project was the Antarctic Syowa Station (Figure A12), which is managed by the National Institute 


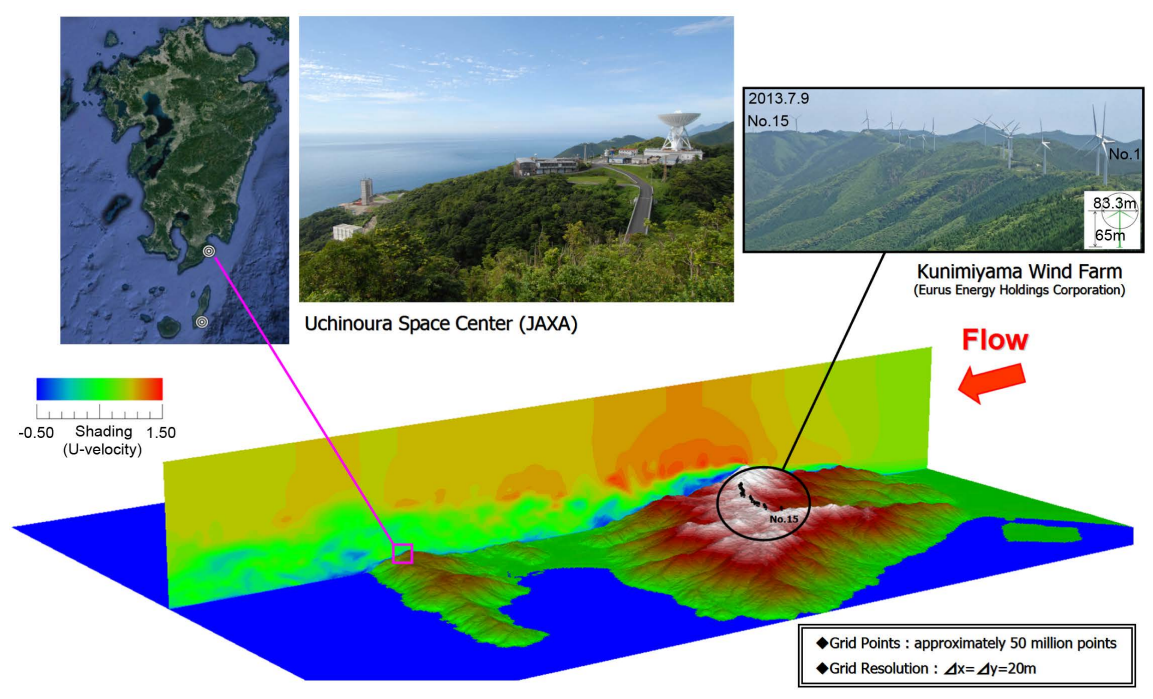

Figure A8. Flow visualization in the numerical simulation in the vertical plane, instantaneous flow field.

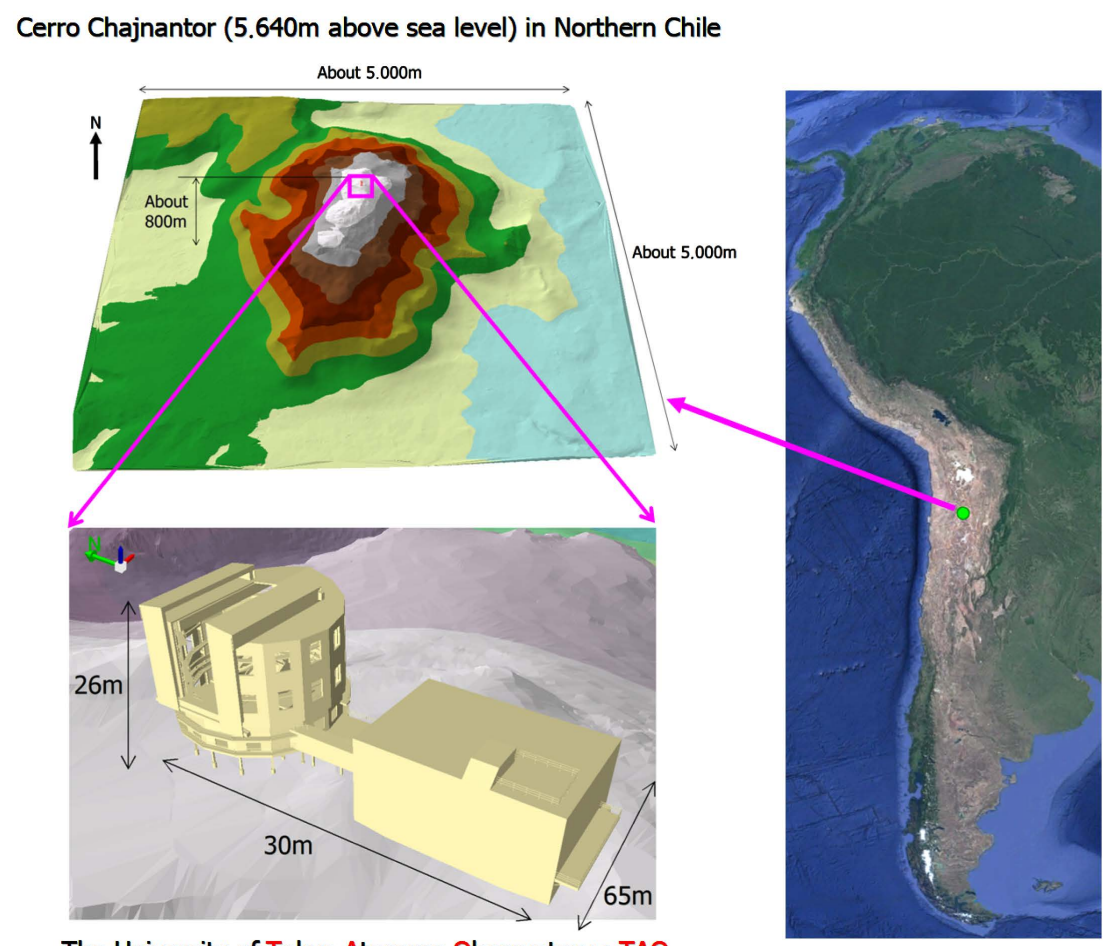

The University of Tokyo Atacama Observatory : TAO

Figure A9. 3D model of Tokyo Atacama Observatory (TAO) and Cerro Chajnantor (5,640 $\mathrm{m}$ above sea level) in Northern Chile.

of Polar Research. This Japanese observation base is located on East Ongul Island, Antarctica, and has a serious problem with snow drift. Massive snow drift accumulated in the administrative building cluster on the base, which had been reformed from 1992 to 2001. This was a serious problem for the management of the Antarctic Syowa Station. Since understanding the characteristics of snow drift is important for implementing control measures, a CFD simulation with a 


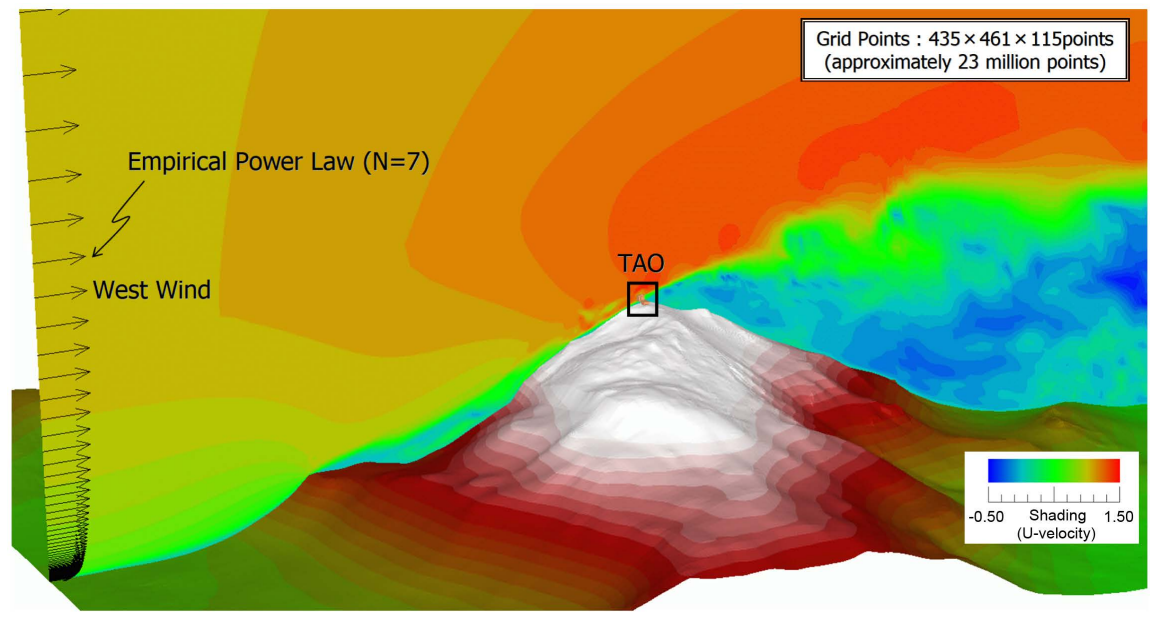

Figure A10. Flow visualization in the numerical simulation in the vertical plane, instantaneous flow field.

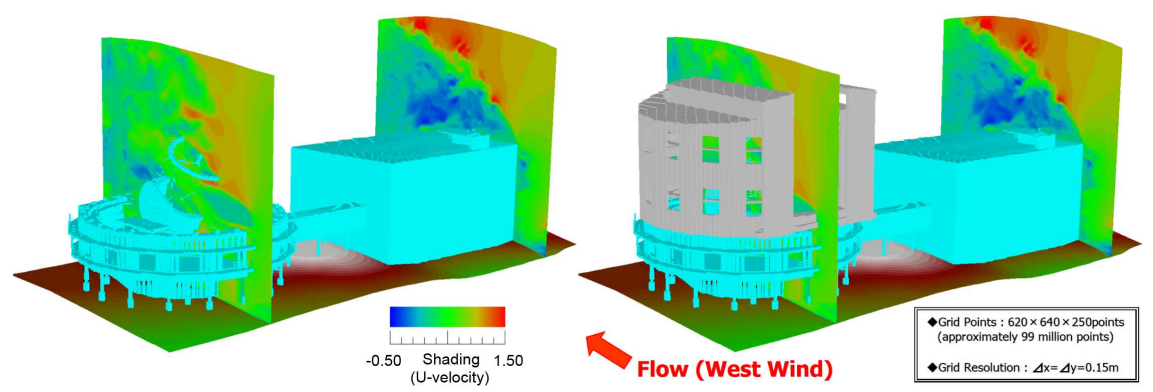

Figure A11. Flow visualization in the numerical simulation in the vertical plane, instantaneous flow field.

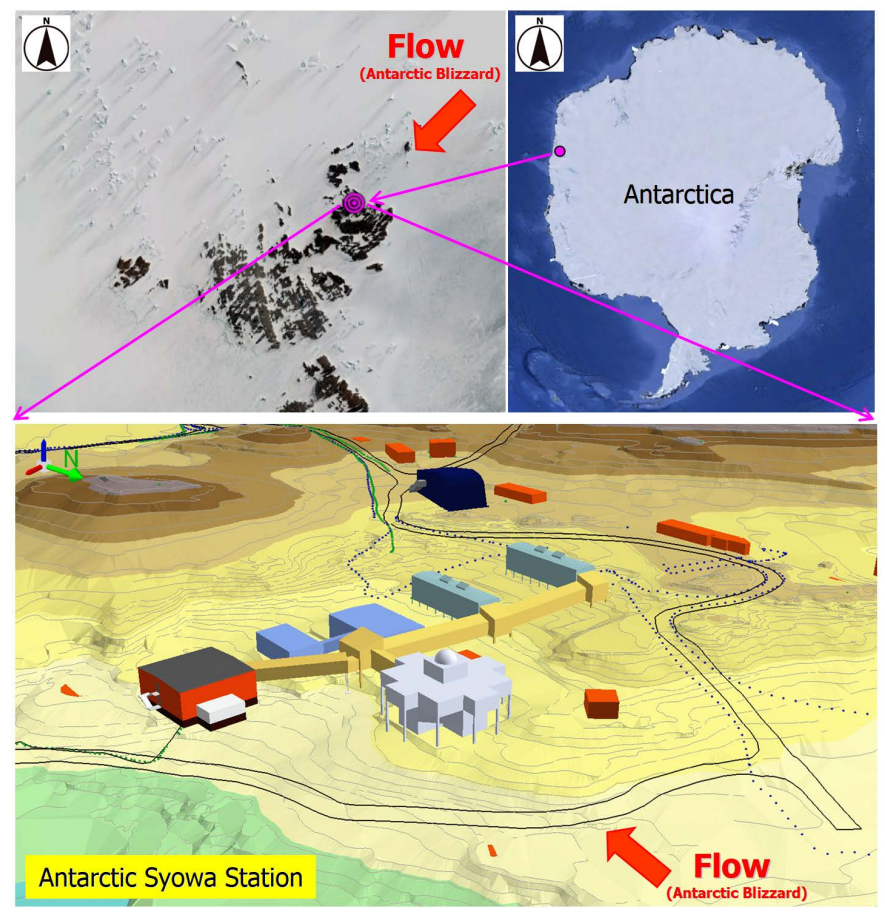

Figure A12. 3D model of Antarctic Syowa Station. 
large number of grid points/cells at a high-resolution was conducted, with the main purpose of establishing the efficient management of construction plans for the Antarctic Syowa Station. Employing Airflow Analyst, the entire Antarctic Syowa Station was simulated to assess the air flow field. The management building cluster and other buildings of the Antarctic Syowa Station were reproduced using a northeast wind with a 0.2 meter spatial resolution (Figure A13). This reproduction clarified how the complexity of air flows, which were generated in the region of the Antarctic Syowa Station and the management building cluster, change depending on time and space (Figure A14 and Figure A15). As a old

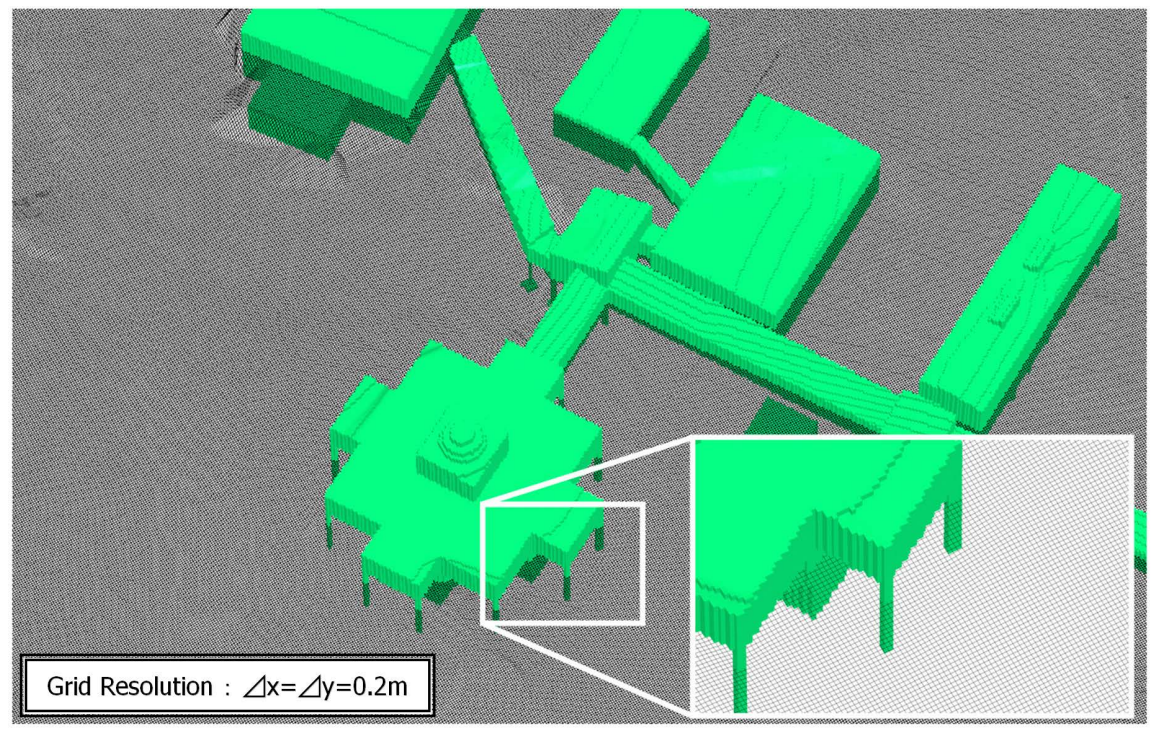

Figure A13. Computational grids in the horizontal plane.

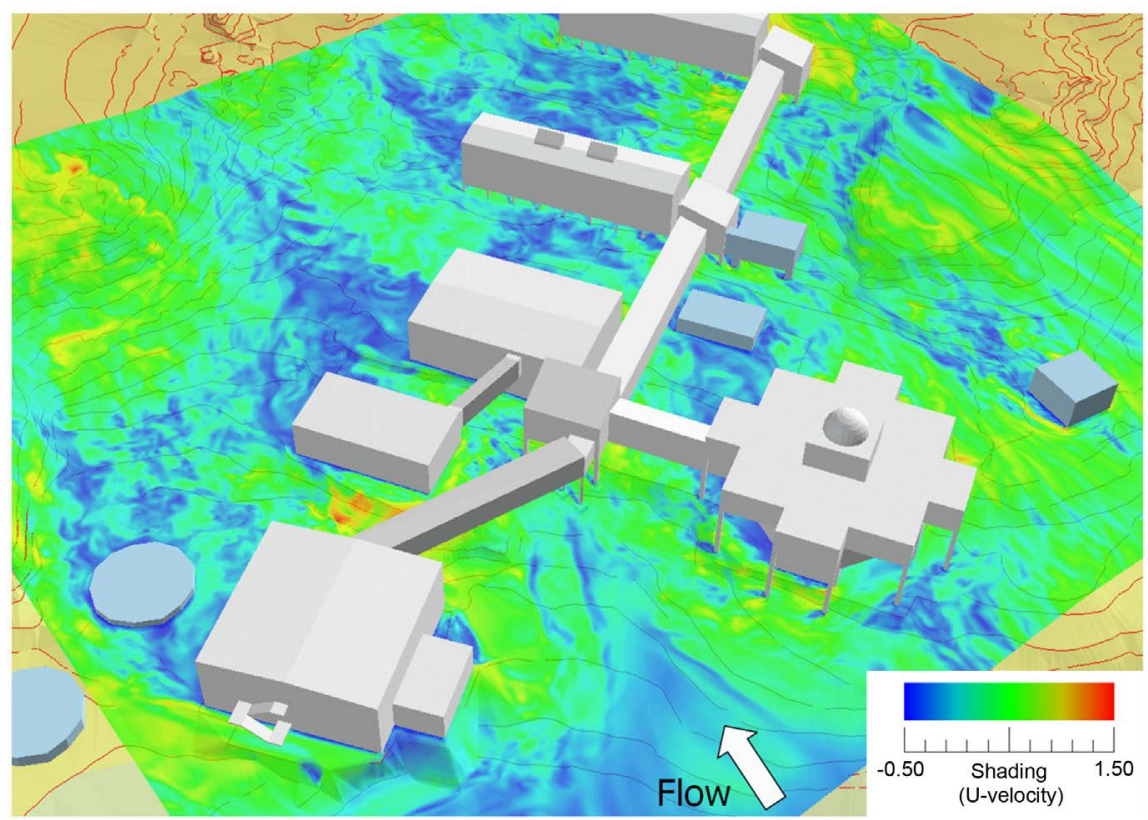

Figure A14. Flow visualization in the numerical simulation near the ground, instantaneous flow field. 


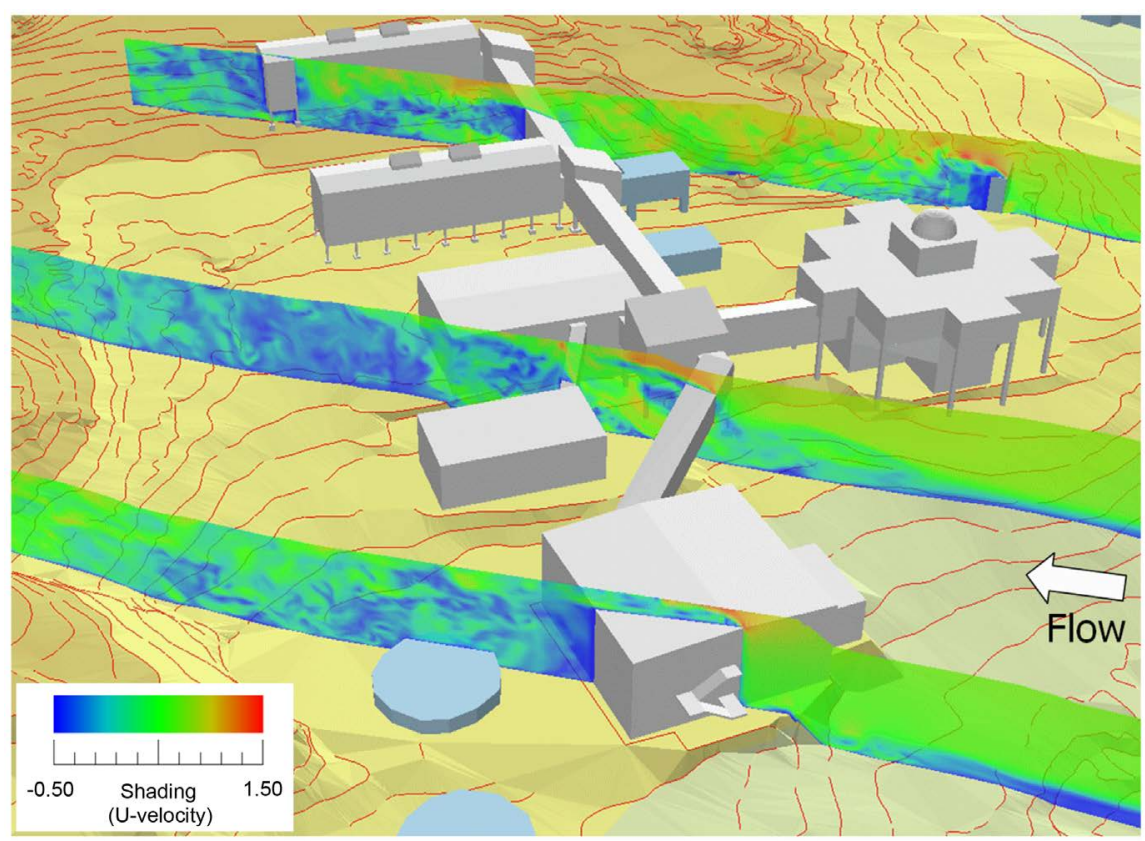

Figure A15. Flow visualization in the numerical simulation in the vertical plane, instantaneous flow field.

saying from Antarctica suggests, "Who controls the wind is who controls Antarctica". The findings of our joint investigation contribute to producing more efficient construction plans for the Antarctic Syowa Station. 